\title{
Debate on Bioavailability and Bioequivalence (BA/BE) Studies Using Lim- ited Sampling Strategy: Views and Perspectives
}

\author{
Nuggehally R Srinivas* \\ Zydus Research Centre, Sarkhej-Bavla NH 8A, Moraiya, Ahmedabad 382210, Gujarat, India
}

The oral bioavailability/bioequivalence (BA/BE) studies serve the purpose of establishing equivalence by comparing treatment versus the appropriate reference product using the pharmacokinetic data of the parent drug (and/or metabolite) as a surrogate. BA/BE studies aim to measure both rate of absorption and extent of absorption of the drug in order to verify whether or not equivalence exists when treatment versus reference product comparison is performed. The absorption rate is linked to the attainment of the peak plasma/serum concentration $\left(\mathrm{C}_{\max }\right)$ and the extent of absorption is measured using area under the plasma/ serum concentration vs. time data up to last time point " $\mathrm{t}$ " ( $\left.\mathrm{AUC}_{0-\mathrm{t}}\right)$ or $\mathrm{AUC}_{0-\mathrm{t}}$ extrapolated up to time infinity $\left(\mathrm{AUC}_{\mathrm{inf}}\right)$. Instead of a single dose, if $\mathrm{BA} / \mathrm{BE}$ studies evaluate steady state concentrations of the two products, the bioequivalence assessment of the extent of absorption is performed using $\mathrm{AUC}_{\text {tau }}$. Both pharmacokinetic surrogates, $\mathrm{C}_{\max }$ and $\mathrm{AUC}_{\text {inf }}\left(\mathrm{AUV}_{0-\mathrm{t}}\right.$ or $\mathrm{AUC}_{\text {tau }}$ as the case may be) are considered for establishing bioequivalence of the test product with that of the reference product. In a typical bioequivalence assessment, both $\mathrm{C}_{\max }$ and $\mathrm{AUC}_{\text {inf }}$ for the two products will be log transformed. The $90 \%$ confidence interval of the relative mean (Geometric mean) of the test to reference products for Ln-transformed $\mathrm{C}_{\text {max }}$ and $\mathrm{AUC}_{\text {inf }}$ should be contained within $80.00 \%$ to $125.00 \%$. In some situations, if the intra-subject variability is very high, the regulators may allow a wider bioequivalence acceptance for $\mathrm{C}_{\max }$ parameter only; however, such cases of widening the confidence interval need to be well-justified.

Because of the importance to accurately pin-point the $\mathrm{C}_{\max }$ of the products, it is imperative to collect as many time points as feasible, around the time where $\mathrm{C}_{\max }$ would generally occur. For instance, if average time of occurrence of $\mathrm{C}_{\max }$ is $2 \mathrm{~h}$ after oral dosing; it may be reasonable to collect frequent pharmacokinetic samples at $0.25 \mathrm{~h}$ time intervals starting from 1 to $3 \mathrm{~h}$ after the oral dosing. By doing a rigorous sample collection, the $\mathrm{C}_{\max }$ value will be more reliable.

In order to get an unambiguous estimation of $\mathrm{AUC}_{\mathrm{inf}}$ of the products, it may be important to extend the collection of pharmacokinetic samples up to 5 half-life values of the drug in question. For instance, if the halflife of the drug is $12 \mathrm{~h}$, it may be reasonable to collect samples up to 60 or $72 \mathrm{~h}$ post oral dosing of the drug. Another rule of thumb in estimating $\mathrm{AUC}_{\text {inf }}$ is to ensure that the area under the tail from the last measurable time point is well below $20 \%$ of the total $\mathrm{AUC}_{\text {inf }}$ (i.e., $\mathrm{AUC}_{0-\mathrm{t}} / \mathrm{AUC}_{\mathrm{inf}}$ $>0.80$ ). Hence, the importance of extending pharmacokinetic samples for up to 5 half-lives becomes a critical consideration.

Given the rigour employed which has been delineated in the preceding paragraphs and the current regulatory expectations in the determination of accurate $\mathrm{C}_{\max }$ and $\mathrm{AUC}_{\text {inf }}$ for the assessment of $\mathrm{BABE}$, the concept of limited sampling strategy for establishing BABE has been introduced in published scientific literature [1-3]. This editorial is intended to provide some perspectives on this evolving concept.

The concept of limited sampling strategy largely relies on establishing a-priori few pharmacokinetic time points, using appropriate statistical methods and validations, that would accurately predict the exposure (i.e. $\mathrm{C}_{\max }$ and $\mathrm{AUC}$ ) of the drug in question using a modelling approach (linear regression model for instance) as enumerated in the following two case studies.

Pidotimod: Huang et al., carried out a 2-way randomized crossover BABE study in healthy human subjects for the evaluation of two formulations of containing $800 \mathrm{mg}$ dose of pidotimod [1]. The pharmacokinetics samples collected in the study were typical of a regular standard protocol for accurately assessing $\mathrm{C}_{\max }$ and $\mathrm{AUC}_{0-10 \mathrm{~h}}$ data. In addition, Huang et al., developed and validated a limited sampling model for correlating $\mathrm{C}_{\max }$ and $\mathrm{AUC}_{0-12 \mathrm{~h}}$ obtained from the study with either 2 to 4 concentration time points to generate linear regression equations for accurately predicting $\mathrm{C}_{\max }$ and $\mathrm{AUC}_{0-12}$ data of pidotimod [1]. The developed linear regression model for prediction of exposure was subjected to internal validation by the Jack-Knife method and the plots were critically evaluated graphically. Both $\mathrm{C}_{\max }$ and $\mathrm{AUC}_{0-12 \mathrm{~h}}$ from the traditional approach were compared with the respective parameter values obtained from the limited sampling method [1]. The findings of this study suggested that limited sampling methods can be readily applied for the prediction of $\mathrm{C}_{\max }$ and $\mathrm{AUC}_{0-12 \mathrm{~h}}$ of pidotimod. Furthermore, limited sampling methods data showed bioequivalence of the test with that of the reference formulation suggesting that a lengthy and more resource intensive collection of samples were not necessary [1].

Metformin: Chen et al., performed a 2-way single dose randomized crossover BABE study of metformin evaluating test versus reference formulation at a dose of $1000 \mathrm{mg}$ in healthy human subjects [2]. The study collected serial pharmacokinetic samples for defining $\mathrm{C}_{\text {max }}$ and an unambiguous estimation of $\mathrm{AUC}_{0-24 \mathrm{~h}}$. By using multiple linear regression analysis, the observed values of $\mathrm{C}_{\max }$ and $\mathrm{AUC}_{0-24 \mathrm{~h}}$ were correlated with one, two or three concentration points of the reference formulation to develop limited sampling models. The models were assessed for accuracy in prediction by the Jack-Knife method. The best model was used to compute the parameters for bioequivalence testing of metformin formulations. Using Jack-Knife method, it was established that 1.5 and $2 \mathrm{~h}$ samples predicted accurate $\mathrm{C}_{\max }$ estimation of metformin. Similarly, 4 and $10 \mathrm{~h}$ samples predicted $\mathrm{AUC}_{0-24 \mathrm{~h}}$ accurately. The bioequivalence assessment (i.e., T/R geometric mean estimate; $90 \%$ confidence intervals) using limited sampling methods were comparable to that obtained from all the pharmacokinetic samples obtained in the

*Corresponding author: Nuggehally R Srinivas, PhD, FCP, Zydus Research Centre, Sarkhej-Bavla NH 8A, Moraiya, Ahmedabad 382210, Gujarat, India, Tel: 918066628801; E-mail: nuggehally.srinivas@zyduscadila.com

Received December 22, 2017; Accepted December 26, 2017; Published January 02, 2018

Citation: Srinivas NR (2018) Debate on Bioavailability and Bioequivalence (BA/ BE) Studies Using Limited Sampling Strategy: Views and Perspectives. J Bioequiv Availab 10: e82. doi:10.4172/jbb.10000e82

Copyright: @ 2018 Srinivas NR. This is an open-access article distributed under the terms of the Creative Commons Attribution License, which permits unrestricted use, distribution, and reproduction in any medium, provided the original author and source are credited. 
study. The authors suggested that the use of limited sampling method was a cheaper alternative as it would avoid costs from sample analysis and/or acquisition of samples.

In an earlier work, Mahmood and Mahayani showed the applicability of the limited sampling model for two drugs that showed 3 -fold differences in half-life values; where one drug had a half-life of $12 \mathrm{~h}$, the other one had a longer half-life of $>35 \mathrm{~h}$ [3].

The above cited examples show the promise of this concept where bioequivalence was concluded using limited sampling models for metformin or pidotimod. Some advantages of this concept include: 1) less housing time of the subjects in the clinical facility especially for drugs with long half-life value; 2 ) less procedures during study conduct including reduced blood sample collection; 3) reduced analytical time and cost of the project. On the contrary, in order to develop the limited sampling model, one has to embark on a complete BA/ BE study (i.e., applying a standard protocol and rigorous collection of all pharmacokinetic sample points). Moreover, the developed limited sampling method may be specific to the institution where the model was developed because, hitherto; there are no published data that suggest the universal applicability of the limited sampling model. Although the two data sets representing metformin and pidotimod used in the establishment of the model appeared to be very robust, it is still largely unknown as to how a limited sampling model would work for drug product(s) that exhibit high inter- and intra-subject variability. In the same context, genetic polymorphism in both metabolizing enzymes and drug transporters which are known to have an effect on the pharmacokinetics of certain drugs, may have an impact on the developed model [4-7].

From a regulatory perspective it is important to convince the regulators that the use of limited sampling models is not a compromise to the original approach of establishing bioequivalence of drug products. It is equally important to unequivocally assure the regulators that there is a low probability of missing the true population $\mathrm{C}_{\max }$ and/ or AUC parameter value by the limited sampling model. From the ensuing discussion, it is also necessary to introspect whether or not a bioequivalent test formulation using limited sampling method is capable of producing similar efficacy and safety as that of the innovator (reference) product.

Although the use of limited sampling strategy has been successfully employed in therapeutic drug monitoring (TDM) of narrow therapeutic index drugs [8-11], it is not the same situation in the case of application of the concept to a BA/BE study. While the application of limited sampling strategy in TDM is specifically applied for safety and efficacy assessment at an individual patient level undergoing clinical therapy, the use of the same concept in BA/BE study is generally in healthy subjects for extrapolation of data to a population level.

It is the opinion of the author that a general consensus should be developed on the applicability of limited sampling models for BABE studies with defined statistical approaches using a wider audience from academia, pharmaceutical industry and regulatory body. Because of the availability of the large data sets for scores of generic versus innovator drugs (ANDA approvals), it may be possible to develop, validate such limited sampling models. The ensuing bioequivalence assessment across wide therapeutic category comprising of all BCS class drugs should be compiled. Such analysis in the near future may pave the way to develop a specific framework and global acceptability on the use of limited sampling strategy for BA/BE studies for regulatory approvals.

\section{Conflict of Interest}

The author has no conflict of interest to report on the contents of the editorial. The editorial is intended to provide views on a topic of interest in the field of bioequivalence/bioavailability of drug products.

\section{References}

1. Chen LF, Jiao JJ, Zhang CL, Lou JS, Liu CX (2011) Bioequivalence assessment of metformin hydrochloride using a limited sampling strategy. Int $\mathrm{J}$ Clin Pharmacol Ther 49: 629-636.

2. Huang JH, Huang XH, Wang K, Li JC, Xie XF, et al. (2013) Bioequivalence evaluation of two formulations of pidotimod using a limited sampling strategy. Biomed Pharmacother 67: 475-480.

3. Mahmood I, Mahayni H (1999) A limited sampling approach in bioequivalence studies: application to long half-life drugs and replicate design studies. Int J Clin Pharmacol Ther 37: 275-281.

4. Xiang Q, Li C, Zhao X, Cui YM (2017) The influence of CYP3A5 3 and BCRPC421A genetic polymorphisms on the pharmacokinetics of felodipine in healthy Chinese volunteers. J Clin Pharm Ther 42: 345-349.

5. Taghavi T, St Helen G, Benowitz NL, Tyndale RF (2017) Effect of UGT2B10 UGT2B17, FMO3, and OCT2 genetic variation on nicotine and cotinine pharmacokinetics and smoking in African Americans. Pharmacogenet Genomics 27: 143-154.

6. Kandasamy M, Tripathy K, Ravi S, Kamath N, Pai B, et al. (2010) Unsuspected poor metabolizer phenotypes of fluoxetine in bioavailability/bioequivalence studies from an indian population perspective. Retrospective pharmacokinetic data evaluation. Arzneimittelforschung 60: 12-21.

7. Kandasamy M, Srinivas P, Subramaniam K, Ravi S, John J, et al. (2010) Differential outcomes from metabolic ratios in the identification of CYP2D6 phenotypes--focus on venlafaxine and O-desmethylvenlafaxine. Eur J Clin Pharmacol 66: 879-887.

8. Kiang TK, Ensom MH (2016) Therapeutic drug monitoring of mycophenolate in adult solid organ transplant patients: an update. Expert Opin Drug Metab Toxicol 12: 545-553.

9. Lee JJ, Beumer JH, Chu E (2016) Therapeutic drug monitoring of 5-fluorouracil. Cancer Chemother Pharmacol 78: 447-464.

10. Andrews LM, Li Y, De Winter BCM, Shi YY, Baan CC, et al. (2017) Pharmacokinetic considerations related to therapeutic drug monitoring of tacrolimus in kidney transplant patients. Expert Opin Drug Metab Toxicol 13: 1225-1236.

11. Myers AL, Kawedia JD, Champlin RE, Kramer MA, Nieto $Y$, et al. (2017) Clarifying busulfan metabolism and drug interactions to support new therapeutic drug monitoring strategies: a comprehensive review. Expert Opin Drug Metab Toxicol. 13: 901-923. 